\title{
A Simple Model for Gliding and Low-Amplitude Flapping Flight of a Bio-Inspired UAV
}

\author{
A. Martín-Alcántara ${ }^{1}$, P. Grau ${ }^{1}$, R. Fernandez-Feria ${ }^{2}$ and A. Ollero ${ }^{1}$
}

\begin{abstract}
Inspired by the efficiency of soaring birds in crossing very large distances with barely flap their wings, this work presents a simple model of UAV that, adopting the capabilites of these animals, could improve the existent multi-rotor devices, not only in efficiency but also in safety and accessibility. Thus, simple analytical approximations to reproduce the behavior of flapping wings UAVs are explored, expecting their integration in on-board CPUs to be solved in real-time flight episodes. A comparison between gliding and wing flapping with these models indicates that the thrust generated by wingstrokes should be controlled in further studies in order to mitigate the oscillations along the path of the vehicle. The geometric parameters of the ornithopter are found to be decisive in this sense, so special attention should be paid during the design stage.
\end{abstract}

\section{INTRODUCTION}

Animal flight is known to be one of the most efficient ways of aerial locomotion. Millions of years of evolution have profiled the flight techniques of birds and insects to improve their efficiency in the air [1]-[3]. Many of them exhibit intermittent active (i.e. flapping) and passive (i.e. gliding or soaring) configurations for energy saving allowing them to travel very large distances with a minimal energetic cost [4]: when birds reach a certain velocity they simply extend their wings and remain in the air while descending steadily. The rate of gliding depends principally on the specie, and it is characterized by the glide ratio quantity $L / D$, which is typically about $10: 1$ to $15: 1$ in most of the birds, reaching even $24: 1$ in albatrosses. These high rates are achieved at very low wing loadings [5].

Nowadays, almost all the autonomous flying vehicles have multiple pairs of rotors for a wide range of applications including not only sensing but also physical interactions and manipulation such as in AEROARMS [6]. Unfortunately, these vehicles are very limited in terms of flight endurance, which are only a few minutes in many cases, in addition to the inherent limitations when interacting with people or operating in ATEX (ATmosphères EXplosives) environments. A more efficient alternative to rotor propellers is the use of wings, that can flap or maintain a fixed angle for gliding, which would mean significant energy saving [7]. There are several other situations for which flapping has been proved

\footnotetext{
*This work was supported by the European Project GRIFFIN ERC Advanced Grant 2017, Action 788247

${ }^{1}$ Robotics, Vision and Control Group, University of Seville, Camino de los Descubrimientos s/n 41092 Seville, Spain amartin34@us.es, pgraudus.es, aollerodus.es

${ }^{2}$ Fluid Mechanics, Andalucía Tech., University of Málaga Dr. Ortiz Ramos s/n, 29071 Málaga, Spain ramon. fernandez@uma.es
}

to be more energetically efficient than rotary wings flight for small UAVs [8].

Therefore, in the last few years, the study of animal flapping flight has aroused an enormous interest motivated by the application of the aerodynamics mechanisms of some species to the design of UAVs [9], [10]. For instance, [11] studied the equations of motion for a birdlike ornithopter from an energetic perspective by using a quasi-steady thinairfoil aerodynamic model. Later, [12] provided simple aerodynamic models of a MAV by using flight test data, and more recently [13] developed a tailless aerial vehicle with a stroke-plane modulation mechanism by which the vehicle can maneuver by using only its wings.

In this sense, wing design is one of the most important aspects in the development of an efficient flapping wings UAV because it fixes the gliding performance of the vehicle in balance with its weight. Following [14] and [15], two well-differentiated types of soaring wings can be found, which allow exploitation in different situations. For instance, long soaring wings of birds cruising the seas, e.g. gulls and albatrosses, present a small induced drag coefficient leading the bird to fly at very small gliding angles. Short wings, instead, allow the vultures and birds of prey to take-off from the ground by flapping energically in the absence of wind [5]. In general, wing shapes and sizes should be designed to fit the performance requirements the ornithopters are designed for.

Despite the enormous development of computers in the recent few years, the numerical calculation of the aerodynamic forces by solving Navier-Stokes equations on-board is far away to be a real-time process. Alternatively, analytical approximated solutions within a validity range are much more appropriate because it can provide a more general point of view, gaining a deeper insight in the aerodynamic performance of the UAV. Therefore, the main interest of this work lies in obtaining simple theoretical expressions for gliding flight configuration by only considering longitudinal dynamics. See e.g. [16]-[18] for similar studies ignoring flight maneuvers such as rolling or yawing.

In order to model the aerodynamic forces appearing on the vehicle, potential theory is known to provide good approximations at Reynolds number large enough to neglect viscous effects. This approach is quite simple and accurate for steady-state problems at small angles of attack, but when unsteady effects appear (e.g., in heaving and pitching motions) the calculation of lift and thrust coefficients has to be modeled under some other considerations, e.g. [19][21]. In this case, the application of the Kutta condition to 
a flapping foil is not completely valid and [22] proposed a viscous correction to account for the flow rotation around the trailing edge. Notwithstanding, a simple approximation is desired in order to obtain analytic solutions that may help to understand the flight performance, so the aerodynamic forces will be modeled here following recent improved results from unsteady, linear potential theory [23], [24].

Thus, gliding and low-amplitude flapping mechanisms of a bio-inspired UAV are studied in this work, providing analytical estimations to be used in the design of flapping flight aerial vehicles. The paper is organized as follows. Section II describes the dynamic equations for a simplified UAV by using simple potential models to characterize the aerodynamic forces. In Section III the experimental setup and the estimation of the Lighthill number (i.e., the drag) for the UAV are specified. In Section IV, comparisons between numerical and theoretical results for steady-state gliding flight are given. Additionally, a parametric study is performed in order to select the tail-wing configuration minimizing the gliding angle. Moreover, the effects of wings heaving on the thrust of the aerial vehicle are investigated through the temporal integration of the equations. Finally, conclusions and further works are summarized in Section V.

\section{MATHEMATICAL MODELING}

\section{A. Nondimensional equations}

The flight dynamics of a simplified UAV of finite-span wings is described in this section. The model presented here approximates the fuselage and the (massless) wings by a rigid body satisfying the dimensionless Newton-Euler equations.

Let $\Lambda, \mathcal{H}, \mathcal{M}, \chi$, and $L i$, be the parameters governing the problem (see II-B for their defitinion), the equations modeling the behavior of the ornithopter in the $\xi-\zeta$ plane are (see Fig. 1)

$$
\begin{aligned}
\frac{\mathcal{M}}{R} \frac{\mathrm{d} u}{\mathrm{~d} t}= & {\left[-C_{D i} \cos \beta-C_{L} \sin \beta-L i \cos \beta\right.} \\
& \left.-\Lambda\left(C_{D i t} \cos \beta+C_{L t} \sin \beta\right)\right] U_{b}^{2} \\
& +\sin \theta-\frac{\mathcal{M}}{A} \omega v, \\
\frac{\mathcal{M}}{A R} \frac{\mathrm{d} v}{\mathrm{~d} t}= & {\left[-C_{D i} \sin \beta+C_{L} \cos \beta-L i \sin \beta\right.} \\
& \left.-\Lambda\left(C_{D i t} \sin \beta-C_{L t} \cos \beta\right)\right] U_{b}^{2} \\
& -\cos \theta+\frac{\mathcal{M}}{R} \omega u, \\
\frac{\mathrm{d} \theta}{\mathrm{d} t}= & \omega, \\
\frac{\mathrm{d} \omega}{\mathrm{d} t}= & \chi R\left[-C_{D i} \cos \beta-C_{L} \sin \beta\right. \\
& \left.+\Lambda \mathcal{H}\left(C_{D i t} \cos \beta+C_{L t} \sin \beta\right)\right] U_{b}^{2},
\end{aligned}
$$

with $u$ and $v$ the velocity components, $U_{b}$ its magnitude, $\omega$ the angular velocity, $\theta$ the pitch angle and $\beta=\arctan (v / u)$ the complementary of the sideslip angle. It is convenient to use the $\beta$ - notation to fix the wings position relative to the stroke plane. These definitions are adopted from [25] for a case of insect hovering. The cross terms appearing in (1) and (2) correspond to the decomposition of the translational velocity in the rotating frame. Note that the body is considered as a punctual mass placed on the center of gravity of the UAV and the wings act as massless patches. This assumption is of course valid for gliding configuration in logitudinal flight, but wings inertia should be incorporated to achieve more accurate results in flapping flight. Despite, it is expected that this simplification could reproduce the main physical features of this study.

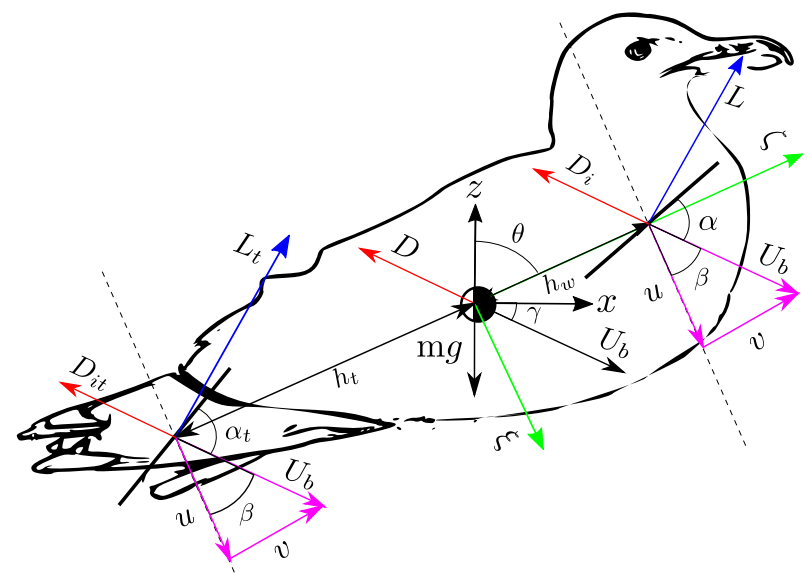

Fig. 1. Schematic of a bio-inspired UAV in forward flight and gliding configurations. The axis $\xi-\zeta$ define a non-inertial reference frame aligned with the dorso-ventral and longitudinal axis, respectively, and corotating with the body according to $\theta$. The wings and tail positions are fixed with $\alpha_{0}=\alpha+\beta$ and $\alpha_{0 t}=\alpha_{t}+\beta$. The aerodynamic forces $L$ and $L_{t}$, and $D_{i}$ and $D_{i t}$, are defined in the conventional form: perpendicular and parallel to the flight velocity, $U_{b}$.

In Fig. 1 the forces acting on the UAV are depicted. The aerodynamic coefficients for the lift $C_{L}, C_{L t}$, and induced drag $C_{D i}, C_{D i t}$, of wings and tail are scaled in the convencional form, i.e. with the dynamic pressure and the corresponding area. From this point on, the subscript $t$ will refer to tail magnitudes.

The scaling of Eqs. (1)-(4) is according to the following relations [26]:

$$
\begin{aligned}
& u \leftarrow u \sqrt{\frac{\rho \bar{c}^{2} R}{2 m g}}, \quad v \leftarrow v \sqrt{\frac{\rho \bar{c}^{2} R}{2 m g}}, \\
& \omega \leftarrow \omega \sqrt{\frac{\rho \bar{c}^{4} R}{2 m g}}, \quad t \leftarrow t \sqrt{\frac{2 m g}{\rho \bar{c}^{4} R}},
\end{aligned}
$$

with $R=b^{2} / A_{w}$ the wing aspect ratio, $\rho$ the air density, $m$ the mass of the UAV, $g$ the gravity acceleration and $\bar{c}=$ $A_{w} / b$ the mean chord length of the wings.

\section{B. Nondimensional parameters}

With the scaling (5), the geometric parameters governing the problem (1)-(4) are the relationship between the tail $A_{t}$ and wing area $A_{w}$, and the ratio between the distances producing moment from the tail $h_{t}$ and from the wing $h_{w}$ to the center of mass of the UAV:

$$
\Lambda=\frac{A_{t}}{A_{w}}, \quad \mathcal{H}=\frac{h_{t}}{h_{w}} .
$$


The rest of parameters involved in (1)-(4) are

$$
\mathcal{M}=\frac{2 m}{\rho \bar{c}^{2}}, \quad \chi=\frac{1}{2} \rho \bar{c}^{4} \frac{h_{w}}{I_{y}}, \quad L i=\frac{A_{b}}{A_{w}} C_{D},
$$

with $\mathcal{M}$ the non-dimensional mass, $\chi$ the dimensionless inverse of the moment of inertia and $L i$ the Lighthill number. The latter represents how the body and propulsor geometry affect the balance of thrust and drag forces on a flier or swimmer of wetted surface $A_{b}$, such that $D=\frac{1}{2} \rho U_{\infty}^{2} A_{b} C_{D}$ [27], [28]. The Lighthill number was estimated from the wind-tunnel experiments of Section III (see below) to be $L i \simeq 0.01$.

In addition, in the flapping flight literature, one can find different parameters relating the stroke motions to the chord length. For instance, in terms of the chord length

$$
k=\frac{\pi f c}{U_{\infty}}=\pi S t_{c}, \quad S t_{c}=\frac{f c}{U_{b}},
$$

or alternatively, based on the wingstroke amplitude

$$
k h_{0}=\frac{\pi f h_{0}}{U_{\infty}}=\frac{\pi}{2} S t_{a}, \quad S t_{a}=\frac{f h_{0}}{U_{b}},
$$

being $f$ the dimensional flapping frequency.

\section{Description of the aerodynamic models}

As the typical chord-based Reynolds number of mediumsized fliers, say $0.5-1 \mathrm{~kg}$, is in the range $50000<R e<$ 100000 (see [29]), the linearized potential theory is expected to be suitable in approximating the aerodynamic performance of a UAV in forward flight. It is demonstrated that natural selection tuned animals for high propulsive efficiency in the range $0.2<S t_{a}<0.4\left(0.31 \lesssim k h_{0} \lesssim 0.63\right)$ and more paticularly $S t_{a} \simeq 0.1(k \simeq 1)$ [see (8)-(9) for the conversion] for gulls [30]. In this narrow band, the work of [23] is appropriate to characterize the unsteady aerodynamic forces of a flapping wing. This recent theory has been compared against other experimental and numerical studies (see e.g. [24], [31]), displaying a good agreement for small wingstroke amplitudes. Note that in the limit of gliding flight, $k h_{0} \rightarrow 0$, the classical potential solution for a flat plate $C_{L}=2 \pi \alpha$ is recovered.

Consequently, the aerodynamic forces generated by the wings and tail of the simplified flier in Fig. 1 are the lift $(L$ and $\left.L_{t}\right)$ and the induced drag $\left(D_{i}\right.$ and $\left.D_{i t}\right)$. For the fuselage, only the parasitic drag $D$ is considered, which is introduced into the equations (1)-(5) through the Lighthill number $\mathrm{Li}$.

In the gliding limit, the lift force developped by the wings of the ornithopter is modeled by usign the well-known potential solution for a finite-span flat plate

$$
C_{L}=2 \pi \alpha \frac{R}{R+2}
$$

where the factor $R /(A+2)$ is due to Prandtl's Lifting-Line Theory for an elliptic aerodynamic loading. On the other hand, following [32], the tail can be considered as a flat delta-wing producing a lift force given by

$$
C_{L t}=\frac{\pi}{2} \alpha_{t} R_{t}
$$

Despite the stall of high aspect ratio wings at angles of attack around $15^{\circ}$, flat triangular plates, like the tail, show an increase in the lift slope after the flow separation and these wings will not suffer stall below angles of attack of $35^{\circ}$. The small slope of lift produced by delta wings compared to that of flat plates ( $\pi / 2$ vs. $2 \pi$ ), makes the tail less sensitive to changes in the angle of attack, providing a more stable lift force under unsteady conditions. This effect is necessary e.g. in flapping flight because the induced velocity generated by wings produces strong fluctuations in the angle of attack of the tail as explained in [33].

Finally, from any of the lift definitions in (10) or (11), the expressions for the induced drag are similarly defined

$$
C_{D i}=\frac{C_{L}^{2}}{\pi R}, \quad C_{D i t}=\frac{C_{L t}^{2}}{\pi R_{t}} .
$$

\section{EXPERIMENTAL ARRANGEMENT}

Experimental tests were performed in the closed-loop, low-speed wind tunnel at the University of Málaga, which has a $4 \mathrm{~m}$ long test section, with one square meter crosssection. See [34] and [35] for technical details about the tunnel.

Figure 2 shows the UAV prototype designed for performing the measurements on the wind tunnel. It is an ornithopter platform with flapping wing capability and orientable tail. Table I summarizes the most significant characteristics regarding the geometry.

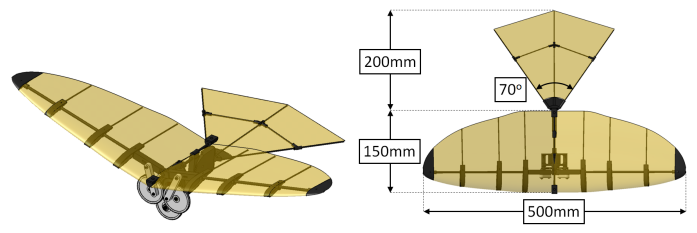

Fig. 2. Schematic of the home-made ornithopter designed for wind tunnel testing.

TABLE I

WING AND TAIL DIMENSIONS

\begin{tabular}{|c|c|c|}
\hline & Wing & Tail \\
\hline Surface $\left(\mathrm{cm}^{2}\right)$ & 590 & 230 \\
\hline Max span $(\mathrm{mm})$ & 500 & 223 \\
\hline Max chord $(\mathrm{mm})$ & 150 & 200 \\
\hline 2D Section & Airfoil & Planar \\
\hline
\end{tabular}

The curvature and the thickness of the wing cross section can be seen in Fig. 3. This shape is given by 3D-printed ribs and carbon fiber rods to avoid an excessive weight. All the ribs are covered with a nylon ripston fabric, tense enough so that it maintains its shape under an incident current. The plant view is elliptical in the trailing edge, and the leading edge presents some curvature derivated from aligning all the airfoils by $1 / 4$ of their chord. A carbon fiber rod on each semi-wing aligns all the airfoils, granting spanwise rigidity. The tail is a flat surface with no movable parts. Carbon fiber 
rods are also used to fix the shape. The surface is the same nylon fabric as the wings.

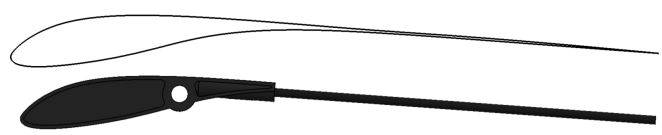

Fig. 3. Airfoil (top) and rib (bottom).

During the tests, the prototype was centered in the test section to avoid wall effects. The force sensor is on the floor of the tunnel, so that a vertical stand is needed to allocate the prototype at a proper height. The stand is $0.5 \mathrm{~m}$ height, and is shown in Fig. 4. It is shaped as a NACA0012 airfoil, so that when aligned with the current there is no flow separation or undesired forces that may alter measurements. The stand is made with 3D printed plastic, with two inner steel rods along the piece to provide more rigidity. Screws in the base fix the stand to the force sensor while at the top the ornithopter is mounted by a gripper.

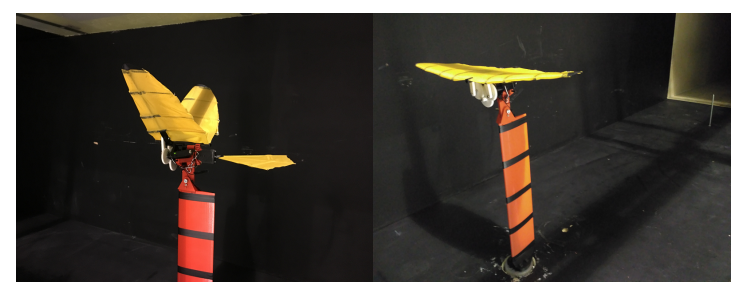

Fig. 4. Experimental setup: Home-made ornithopter mounted inside the wind tunnel.

Several measurements were made for various angles of attack in order to take a drag averaged value for the Lighthill number estimation. The experiments ranged from $0^{\circ}$ to $25^{\circ}$ in steps of $5^{\circ}$.

The Reynolds number of the experiments, based on the mean-chord lenght of the UAV, was estimated to be $R e \simeq$ 50000 , corresponding to a freestream velocity $U_{\infty} \simeq 8 \mathrm{~m} / \mathrm{s}$, in the order of a medium-sized natural glider.

\section{RESULTS AND DISCUSSION}

The present study proposes approximated steady solutions for the case of a simplified UAV in gliding flight. The tailwing conditions for stable gliding as well as those that minimize the gliding angle are explored. The analytic results are endorsed numerically by using the fsolve function in MATLAB (The MathWorks, Inc., Natick, MA, USA). Finally, the Newton-Euler equations in (1)-(4) are integrated numerically with the ode 45 routine to analyze the unsteady effects introduced by the heaving motion of the wings.

\section{A. Steady-state solutions}

The equations (1)-(4) are rewritten in steady form as

$$
\begin{aligned}
0= & {\left[-C_{D i} \cos \beta-C_{L} \sin \beta-L i \cos \beta\right.} \\
& \left.-\Lambda\left(C_{D i t} \cos \beta+C_{L t} \sin \beta\right)\right] U_{b}^{2} \\
& +\sin \theta, \\
0= & -C_{D i} \sin \beta+C_{L} \cos \beta-L i \sin \beta \\
& \left.-\Lambda\left(C_{D i t} \sin \beta-C_{L t} \cos \beta\right)\right] U_{b}^{2} \\
& -\cos \theta, \\
0= & -C_{D i} \cos \beta-C_{L} \sin \beta \\
& +\Lambda \mathcal{H}\left(C_{D i t} \cos \beta+C_{L t} \sin \beta\right),
\end{aligned}
$$

having used $\omega=0$ from (3).

Due to the nonlinearity of (13)-(15), perturbation methods are applied to linearize the system for $\beta$ and $\theta$ according to

$$
\begin{aligned}
& \beta=\frac{\pi}{2}+\beta^{\prime}, \\
& \theta=\frac{\pi}{2}+\theta^{\prime},
\end{aligned}
$$

with $\beta^{\prime}$ and $\theta^{\prime}$ small, and thus obtaining a simplified analytical solution.

From (15),

$$
C_{L}=\Lambda C_{L t}
$$

is obtained immediately. This expression provides a geometric relationship that fixes the angle between the velocity components through

$$
\beta=\frac{\Lambda \mathcal{H} K_{t} \alpha_{0 t}-K \alpha_{0}}{\Lambda \mathcal{H} K_{t}-K},
$$

where, for simplicity, $K_{t}$ and $K$ are the constants derived from $C_{L}$ and $C_{L t}$, respectively,

$$
\begin{aligned}
K & =2 \pi \frac{R}{R+2}, \\
K_{t} & =\frac{\pi}{2} R_{t} .
\end{aligned}
$$

By introducing (18) in (13)-(14), one obtains

$$
\begin{aligned}
v= & {\left[\Lambda K_{t}\left(\alpha_{0 t}-\beta\right)(\mathcal{H}+1)-L i \beta^{\prime}\right]^{-\frac{1}{2}}, } \\
\theta= & \frac{\pi}{2}+\left[\Lambda K_{t}\left(\alpha_{0 t}-\beta\right)(\mathcal{H}+1) \beta^{\prime}\right. \\
& +L i] v^{2}+\theta_{i}^{\prime},
\end{aligned}
$$

with $\theta_{i}^{\prime}$ the term correcting the solution without considering the induced drag $C_{D i}$ and $C_{D i t}$,

$$
\theta_{i}^{\prime}=\frac{K_{t}^{2}}{\pi}\left(\alpha_{0 t}-\beta\right)^{2}\left[\frac{(\Lambda \mathcal{H})^{2}}{R}+\frac{\Lambda}{R_{t}}\right] v^{2} .
$$

The rest of the flight magnitudes can be directly derived from Fig. 1 and the previous expressions,

$$
\begin{aligned}
\alpha & =\alpha_{0}-\beta, \\
\alpha_{t} & =\alpha_{0 t}-\beta, \\
u & =v \tan ^{-1} \beta, \\
\gamma & =\theta-\beta,
\end{aligned}
$$

where $\gamma$ is the so-called gliding angle. 
In (22), it is observed that

$$
\Lambda K_{t}\left(\alpha_{0 t}-\beta\right)(\mathcal{H}+1)-L i \beta^{\prime}>0
$$

must be satisfied to obtain real velocity values. It provides a geometric restriction between $\alpha_{0}$ and $\alpha_{0 t}$ that makes the solution stable:

$$
\alpha_{0 t}<\frac{\frac{\pi}{2} L i+\alpha_{0}(\mathcal{G}-1)\left[\Lambda K_{t}(\mathcal{H}+1)+L i\right]}{\Lambda K_{t}(\mathcal{H}+1)(\mathcal{G}-1)+\mathcal{G} L i},
$$

being $\mathcal{G}$ a geometric parameter defined as

$$
\mathcal{G} \equiv \frac{\Lambda \mathcal{H} K_{t}}{\Lambda \mathcal{H} K_{t}-K}
$$

Note that this condition can be approximately simplified by

$$
\alpha_{0 t} \lesssim \alpha_{0}
$$

because the Lighthill number is in general small compared to the other terms.

Moreover, static stability derivatives from the present model provide some relations that may be of utility in the design of flapping-wings UAVs,

$$
\begin{aligned}
\frac{\partial C_{M}}{\partial \beta} & =K-\Lambda \mathcal{H} K_{t}<0, \\
\frac{\partial C_{L}}{\partial \beta} & =-K<0, \\
\frac{\partial C_{L t}}{\partial \beta} & =-K_{t}<0
\end{aligned}
$$

with

$$
\begin{array}{r}
C_{M}=R\left[-C_{D i} \cos \beta-C_{L} \sin \beta+\right. \\
\\
\left.\Lambda \mathcal{H}\left(C_{D i t} \cos \beta+C_{L t} \sin \beta\right)\right],
\end{array}
$$

the pitching moment coefficient.

Condition (33) yields,

$$
\Lambda \mathcal{H}>\frac{K}{K_{t}} \equiv 4 \frac{R}{R_{t}(R+2)} .
$$

Note that the force expressions (34) and (35) are satisfied inconditionally for being $K$ and $K_{t}$ the positive slopes of the lift coefficients for the wings and tail, respectively.

In order to determine the range of validity of the previous expressions, the steady-state equations (13)-(15) are solved numerically and compared with the above analytical solutions for small values of $\beta^{\prime}$ and $\theta^{\prime}$, considering the parameters of Table II, for the ornithopter prototype in Section II. The values of $\alpha_{0}$ and $\alpha_{0 t}$ ranged from $75^{\circ}$ to $105^{\circ}$, and their influence on $v, \gamma, \theta, \alpha$ and $\alpha_{t}$, has been analyzed. Of course, this study was performed satisfying (32) and (36).

\section{TABLE II}

SUMMARY OF THE VALUE OF THE ORNITHOPTER PARAMETERS.

\begin{tabular}{|c|c|c|c|c|c|c|}
\hline $\mathcal{M}$ & $\Lambda$ & $\mathcal{H}$ & $L i$ & $\chi$ & $\Re$ & $\Re_{t}$ \\
\hline 6 & 0.3 & 8 & 0.01 & 0.075 & 4.6 & 2.3 \\
\hline
\end{tabular}

Figure 5 shows that the theoretical solutions are in general in good agreement with the numerical references, especially when $\alpha_{0 t}$ approximates to $\alpha_{0}$. Note that for the lowest values of $\alpha_{0}$ and $\alpha_{0 t}$ the error is significant but for tail positions above $85^{\circ}$ the differences are negligible.

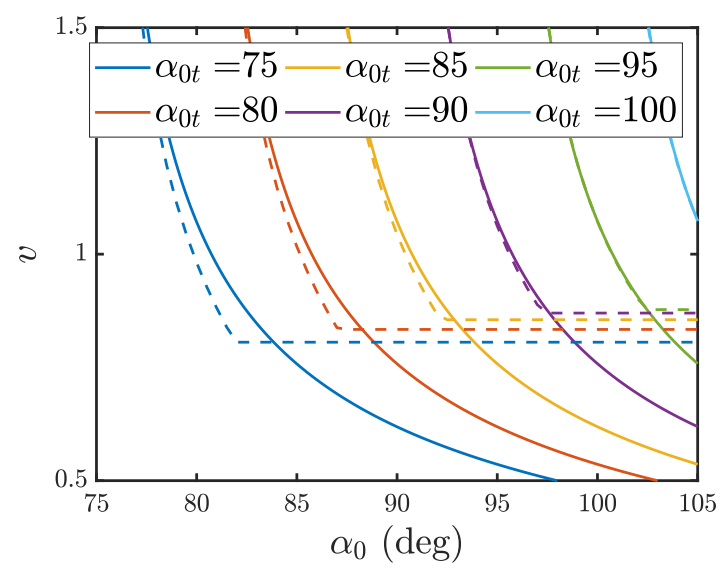

Fig. 5. Comparison of the linear theoretical solutions (solid lines) with nonlinear numerical ones (dashed lines) in terms of $v$. The constant values appearing on the plot are due to the saturation of the lift curves for $\alpha>15^{\circ}$ and $\alpha_{t}>35^{\circ}$ in the numerical solution.

It is also of interest to investigate the effect of the wingtail combinations on the pitch angle, $\theta$. In Fig. 6 one can appreciate again that the theoretical solution is quite accurate when compared with the numerical one. According to that figure, a given value of $\theta$ can be reached from different $\alpha_{0}-\alpha_{0 t}$ relations. For instance, to achieve a typical angle around $90^{\circ}$ (completely horizontal flight), one could select the values $\left(\alpha_{0 t}, \alpha_{0}\right)$ between $\left(85^{\circ}, 85.5^{\circ}\right)$ and $\left(90^{\circ}, 92.8^{\circ}\right)$. No solutions exist out of this range because either the numerical solution becomes singular due to the violation of (33) or the set value is not achieved.

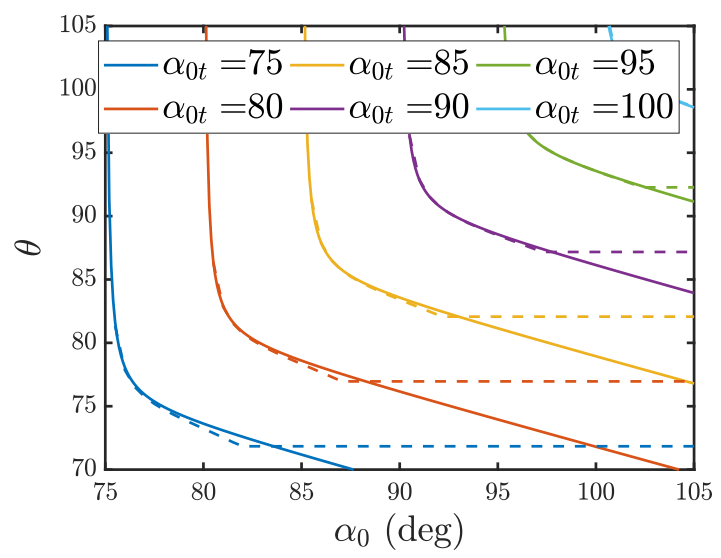

Fig. 6. As in Fig. 5 but for $\theta$.

Figures 7 and 8 show the evolution of the angles of attack in wings and tail, respectively. For this study the lift slopes have been limitated to $\alpha<15^{\circ}$ and $\alpha_{t}<35^{\circ}$, for the reasons stated in Section II-C. This saturation effect is more restrictive here in wings than in tail because of (32). Above these values, the lift curves $C_{L}$ and $C_{L t}$ does not reproduce the expressions (10) or (11) and the results are 
not reliable. Notwithstanding, the theoretical and numerical solutions agree quite well in both figures.

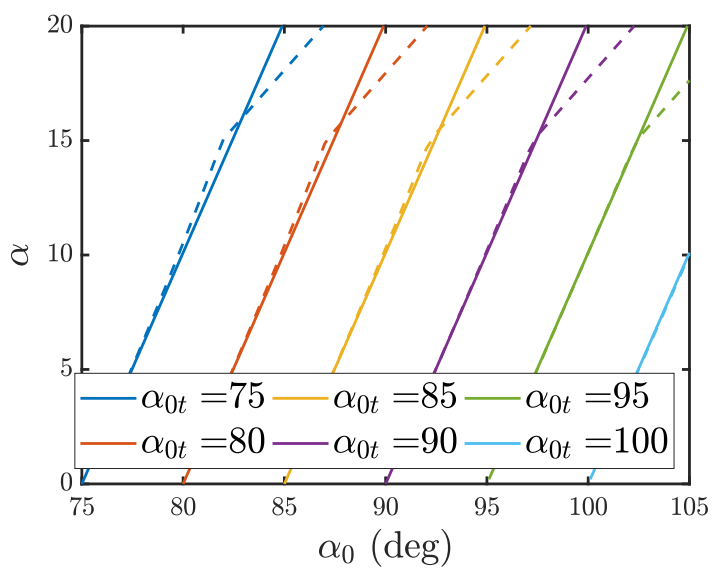

Fig. 7. As in Fig. 5 but for $\alpha$.

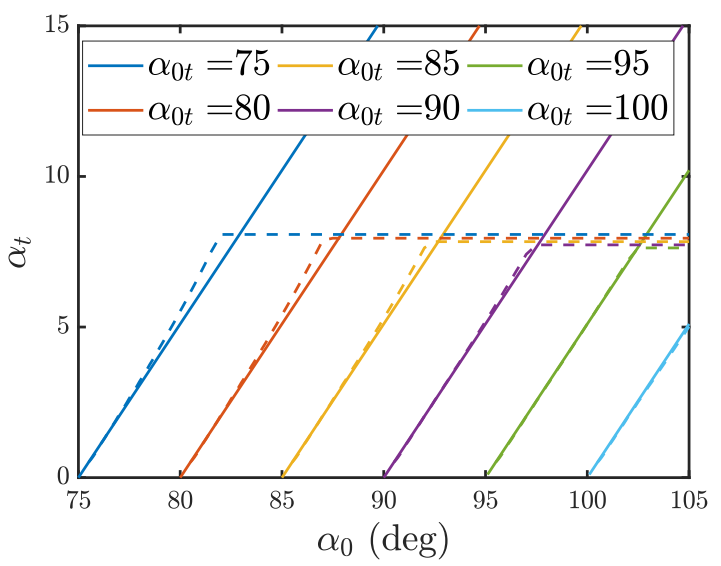

Fig. 8. As in Fig. 5 but for $\alpha_{t}$.

The most meaningful results for gliding flight are plotted in Fig. 9 for $\gamma$, where the theoretical approach practically matches the reference no matter the selected wing-tail configuration. It can be seen that for each $\alpha_{0 t}$ there is always a corresponding value of $\alpha_{0}$ that minimizes the gliding angle. Such minimum value $\gamma_{0}$ will be of course different depending on the geometric parameters of the UAV $\Lambda, \mathcal{H}$ and $L i$, according to (28).

Figure 10 shows the evolution of the minimum gliding angles in the range studied in Fig. 9 with the tail position. Notice that, although the theoretical predictions show the existence of a minimun value of $\gamma_{0}$ at about $\alpha_{0 t}=90^{\circ}$, the numerical results reveal that $\gamma_{0}$ is practically constant along $\alpha_{0 t}$, agreeing quite well both approaches near $\alpha_{0 t}=90^{\circ}$.

More intriguing are the results that relate $\gamma_{0}$ with the flight quantities appearing in Figs. 5-9. For example, Fig. 11 displays the line $\alpha_{0}$ vs. $\alpha_{0 t}$ that minimizes $\gamma$. This figure is of great practical interest for the control of gliding UAVs because, given a wing position, one can find the appropriate tail configuration for the vehicle to descend in as much time as possible, at zero energy cost. This is of critical importance

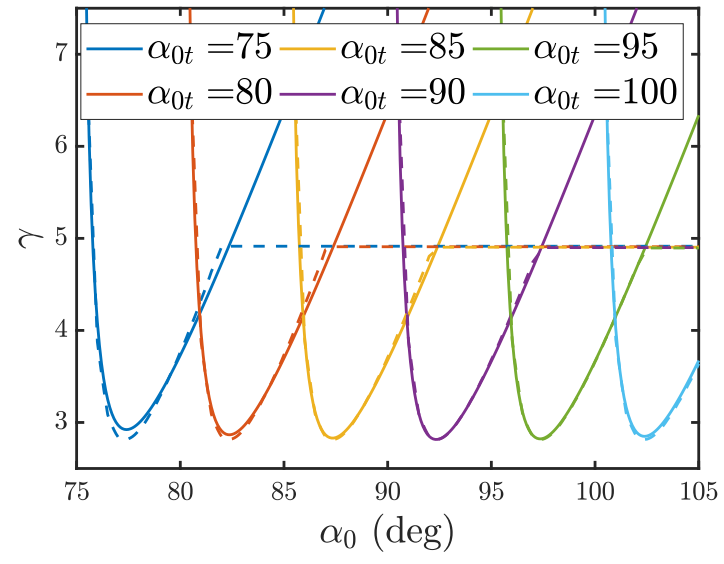

Fig. 9. As in Fig. 5 but for $\gamma$.

to optimize both the weight and size of the vehicle in order to travel large distances more efficiently.

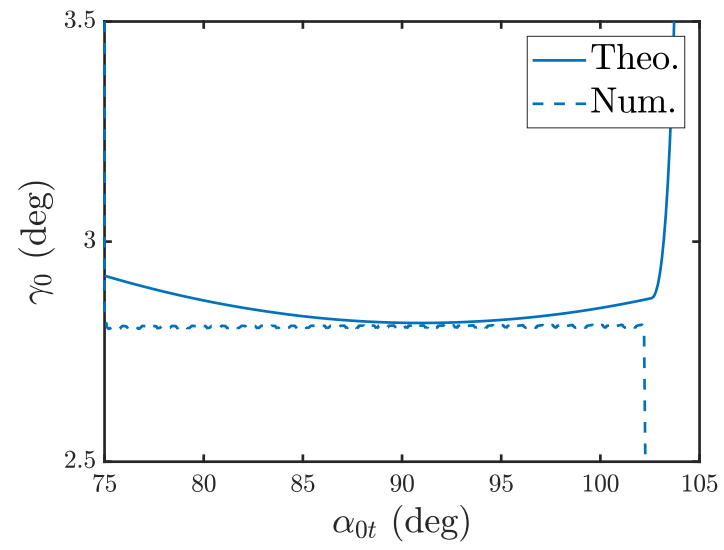

Fig. 10. Evolution of $\alpha_{0 t}$ for the minumum values of the gliding angle $\gamma$.

Another magnitude of interest is the optimum velocity $v$, displayed in Fig. 12. The figure depicts that there exists only a value $v \simeq 1.5$ that minimizes $\gamma_{0}$, regardless of $\alpha_{0}$ and $\alpha_{0 t}$, and depending on the geometric parameters of the UAV. If some other values of $v$ would be desirable, then the parameters of the ornithopter should be adjusted during the design phase. However, it is noticeable that $v$ barely varies with the tail position, remaining practically constant given a set of geometric parameters. Likewise, the optimum angles of attack of wings and tail remain also constant with $\alpha_{0}$ and $\alpha_{0 t}$ (see Figs. 13 and 14), being always sufficiently small to satisfy the potential theory restrictions. On the contrary, the action of the tail has the greatest effect on the optimum value of $\theta$ as shown in Fig. 15.

To conclude, it is worth to mention that obtaining analytic expressions to approximate the optimum gliding variables as a function of $\alpha_{0}$ or $\alpha_{0 t}$ is out of the scope of this work and it is being investigated currently.

\section{B. Unsteady solutions in heaving-wing flight}

In addition to glide, it is also needed that a flapping-wings UAV could stroke efficiently its wings to generate thrust 


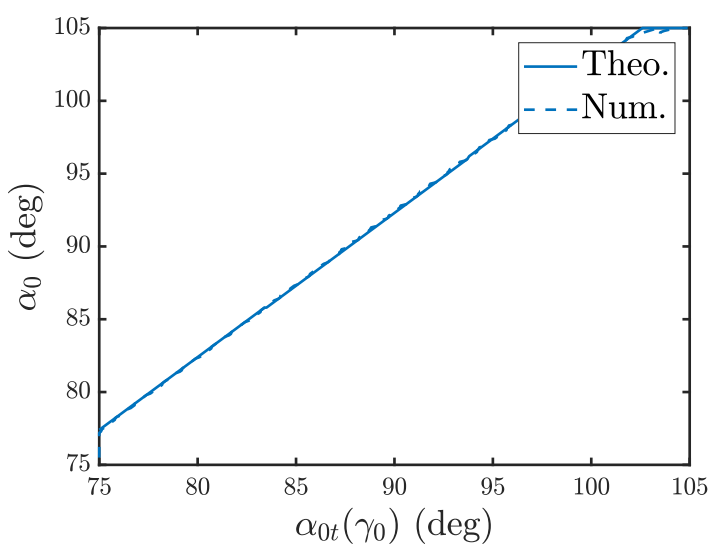

Fig. 11. Relationship for $\alpha_{0}$ and $\alpha_{0 t}$ that minimizes $\gamma$.

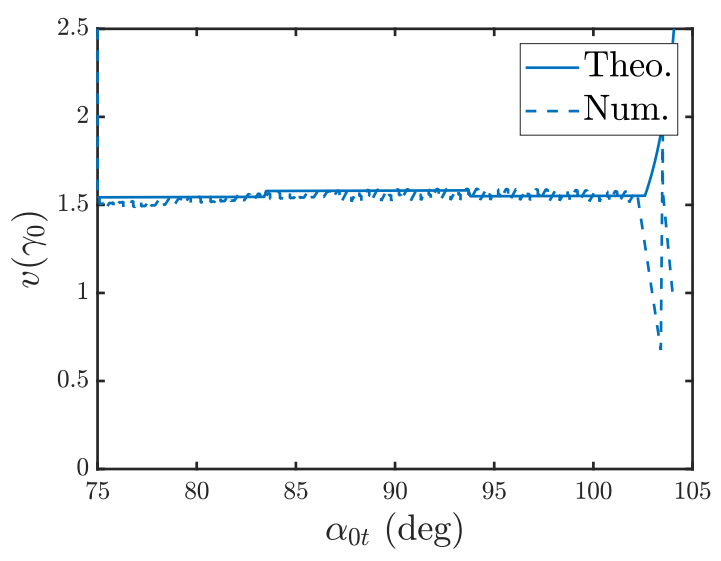

Fig. 12. As in Fig. 8 but for $v$

when needed, e.g. to control the height and alternate both flight configurations to maximize its efficiency.

Although the flapping-wing theory presented in [23], [24], covers both heaving and pitching motions, only the first configuration will be addressed in the current work for the sake of simplicity. The characterization of the flapping motion will be given by appropriate values of $k$ and $k h_{0}$. In order to mimic a realistic case, the flapping parameters were first selected as $k h_{0} \simeq 0.6$ and $h_{0} \simeq 3.8$, following [33] and [36] for typical values in gulls. However, these parameters make the flight unstable for the home-made ornithopter of Fig. 4. To overcome this, the stroke amplitude was decreased to $h_{0} \simeq 1.5$. It also indicates that special attention should be paid to the selection of the geometric parameters of the ornithopter in order to mantain the stability in heaving-wings mode. Therefore, the case plotted in Figs. 16 and 17 has been simulated at an averaged $k h_{0} \simeq 0.15$, with $h_{0}$ fixed to 1.5 .

Figure 16 shows the phase diagram of velocities in heaving-wings flight from the steady-state solution obtained in a gliding configuration. Note that the forward velocity $v$ has been shift towards a higher speed zone in the range $2 \lesssim v \lesssim 2.5$, but the vertical velocity $u$ ranges from negative to positive values, indicating the oscillatory feature of the UAV. It can also be appreciated in Fig. 17 the effect of wings heaving on other magnitudes of interest, e.g. the mean

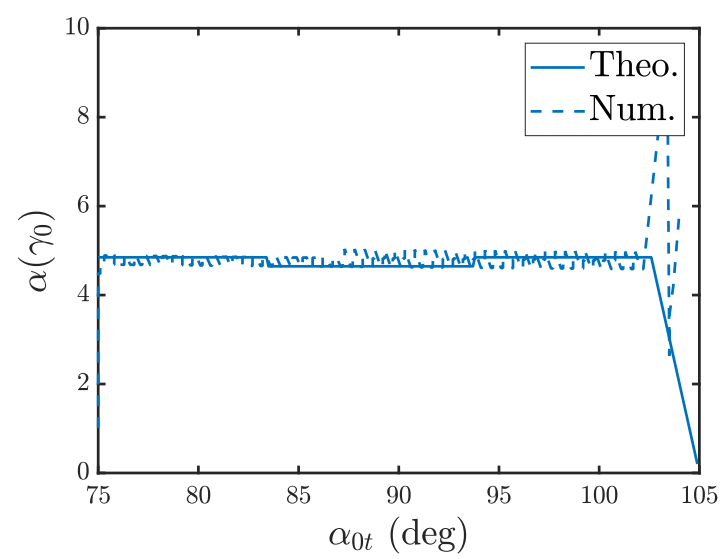

Fig. 13. As in Fig. 2 but for $\alpha$.

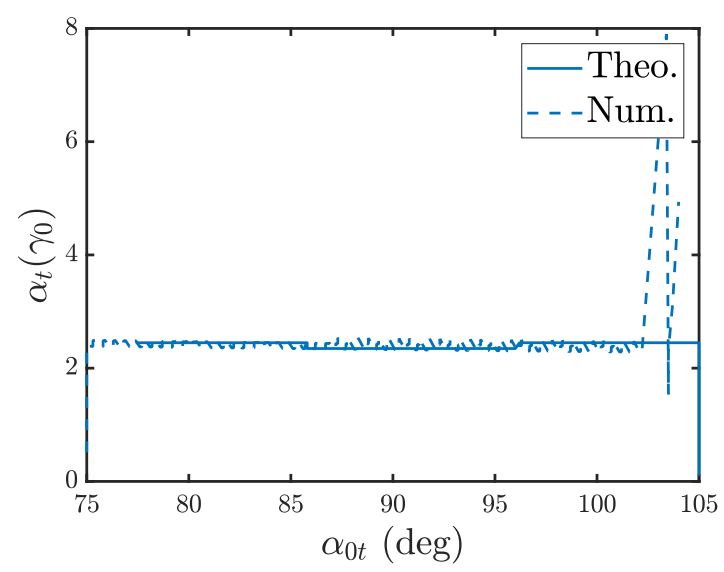

Fig. 14. As in Fig. 2 but for $\alpha_{t}$.

pitch angle, wich is around $69^{\circ}$ in contrast to the practically horizontal attitude $\left(\theta=88^{\circ}\right)$ reached in the steady gliding configuration, or the gliding angle, that presents negative values indicating that the vehicle is climbing. The effect of flapping on the thrust and on the flight path will be addressed in subsequent investigations.

\section{CONCLUSIONS}

Control systems based on complex aerodynamic models requiring numerical solutions are not possible to implement in real time processes with modest computational resources such as on-board CPUs of very light ornithopters, in which the payload is very restricted. Therefore, this work proposes some analytical relations that can be used to control gliding and low-amplitude flapping flight of a bio-inspired UAV.

Thus, under certain simplifications (a punctual mass UAV of massless wings in longitudinal flight), potential aerodynamic models are used to predict the behavior of the birdlike vehicle. For this reason, such elementary approach is only valid for gliding and low-amplitude flapping flight, since separation phenomena appearing in some flight maneuvers are discard. Experimental studies of a home-made ornithopter in a wind tunnel were performed to characterize the parasitic drag of the vehicle in order to obtain realistic results from these models. It is found that theoretical (linear) estimations 


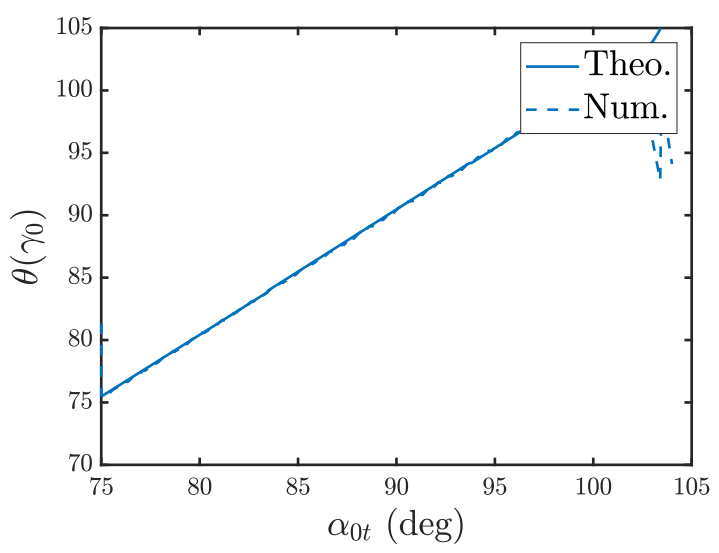

Fig. 15. As in Fig. 2 but for $\theta$

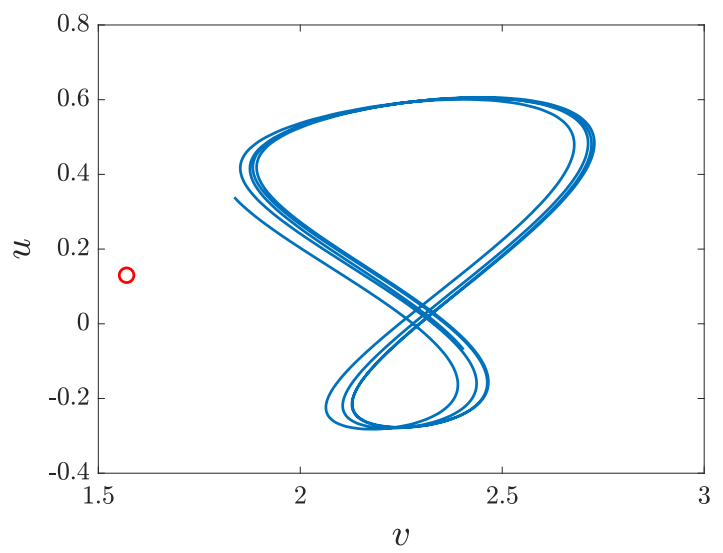

Fig. 16. Phase diagram of the velocity components for $k h_{0} \simeq 0.18$ and $h_{0} \simeq 1.5$. The red circle indicates the steady-state value reached in gliding.

agree quite well with the nonlinear, numerical, steady and unsteady solutions for gliding configuration. It allows to select the tail and wing positions that minimize the gliding angle and so obtain the optimum flight varibles. Moreover, this work explores the effect of flapping (heaving wings) in the reduced model, displaying a monotonic increase in the time-averaged horizontal velocity with $k h_{0}$ (or $\mathrm{St}$ ).

In the latter case, strong oscillations appear on the flight variables when the wingstrokes are introduced, so that a an appropriate control system would be required to mitigate these fluctuations and, in general, to make the flight be stable. For instance, it would be interesting to find the optimum flapping frequency for the ornithopter to maintain a certain height after a gliding episode at the minimum energy cost. This scenario could be the topic of a future research contributing to the development of flapping-wings aerial vehicles.

\section{ACKNOWLEDGMENT}

The authors acknowledge support from the European Project GRIFFIN ERC Advanced Grant 2017, Action 788247. They also wish to thank J. Aguilar-Cabello for his valuable collaboration in performing the wind-tunnel experiments.
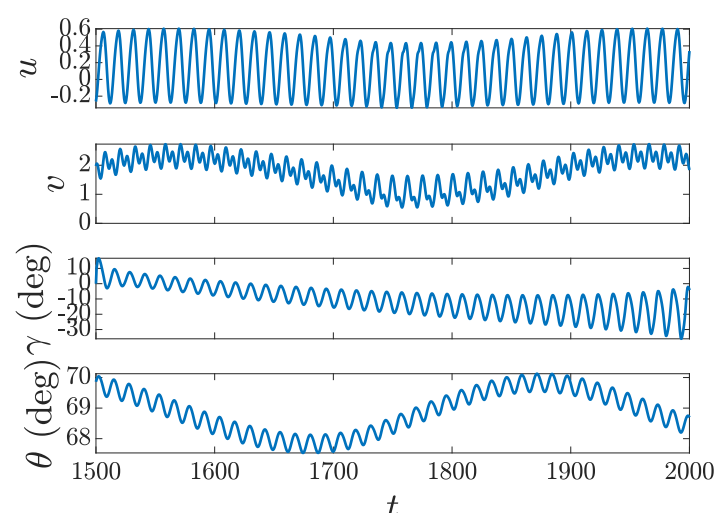

Fig. 17. Temporal evolution of the indicated flight variables for $k h_{0} \simeq$ 0.18 and $h_{0} \simeq 1.5$.

\section{REFERENCES}

[1] R. Dudley, The biomechanics of insect flight: form, function, evolution. Princeton University Press, 2002.

[2] U. M. Norberg, Vertebrate flight: mechanics, physiology, morphology, ecology and evolution, vol. 27. Springer Science \& Business Media, 2012.

[3] R. Dudley and S. P. Yanoviak, "Animal aloft: the origins of aerial behavior and flight," Integrative and comparative biology, vol. 51, no. 6, pp. 926-936, 2011.

[4] B. W. Tobalske, "Biomechanics of bird flight," Journal of Experimental Biology, vol. 210, no. 18, pp. 3135-3146, 2007.

[5] U. M. L. Norberg, "Flight and scaling of flyers in nature," Flow Phenomena in Nature, vol. 1, pp. 120-154, 2007.

[6] A. Ollero, G. Heredia, A. Franchi, G. Antonelli, K. Kondak, A. Sanfeliu, A. Viguria, J. R. Martinez-De Dios, F. Pierri, J. Cortes, A. Santamaria-Navarro, M. A. Trujillo, R. Balachandran, J. AndradeCetto, and A. Rodriguez, "The AEROARMS Project: Aerial Robots with Advanced Manipulation Capabilities for Inspection and Maintenance," IEEE Robotics and Automation Magazine, vol. 25, no. 4, pp. 12-23, 2018.

[7] GRIFFIN, "GRIFFIN ERC Advanced Grant 2017, Action 788247." https://griffin-erc-advanced-grant.eu/, 2019. [Online; accessed 2019/05/10].

[8] M. I. Woods, J. F. Henderson, and G. D. Lock, "Energy requirements for the flight of micro air vehicles," The Aeronautical Journal, vol. 105, no. 1045, pp. 135-149, 2001.

[9] C. P. Ellington, "The novel aerodynamics of insect flight: applications to micro-air vehicles," Journal of Experimental Biology, vol. 202, no. 23, pp. 3439-3448, 1999.

[10] M. F. Platzer, K. D. Jones, J. Young, and J. C. S. Lai, "Flapping wing aerodynamics: progress and challenges," AIAA journal, vol. 46, no. 9, pp. 2136-2149, 2008.

[11] J. A. Grauer and J. E. Hubbard, "Multibody model of an ornithopter," Journal of guidance, control, and dynamics, vol. 32, no. 5, pp. 16751679, 2009

[12] J. V. Caetano, C. De Visser, G. De Croon, B. Remes, C. De Wagter, J. Verboom, and M. Mulder, "Linear aerodynamic model identification of a flapping wing mav based on flight test data," International Journal of Micro Air Vehicles, vol. 5, no. 4, pp. 273-286, 2013.

[13] Q.-V. Nguyen and W. L. Chan, "Development and flight performance of a biologically-inspired tailless flapping-wing micro air vehicle with wing stroke plane modulation," Bioinspiration \& biomimetics, vol. 14, no. 1, p. 016015, 2018.

[14] C. Pennycuick, "Soaring behaviour and performance of some east african birds, observed from a motor-glider," Ibis, vol. 114, no. 2, pp. 178-218, 1972.

[15] C. Pennycuick, "Thermal soaring compared in three dissimilar tropical bird species, fregata magnificens, pelecanus occidentals and coragyps atratus," Journal of Experimental Biology, vol. 102, no. 1, pp. 307325, 1983.

[16] G. Taylor and A. Thomas, "Animal flight dynamics ii. longitudinal stability in flapping flight," Journal of theoretical biology, vol. 214, no. 3, pp. 351-370, 2002. 
[17] F. Gavilan, J. Acosta, and R. Vazquez, "Control of the longitudinal flight dynamics of an uav using adaptive backstepping," IFAC Proceedings Volumes, vol. 44, no. 1, pp. 1892-1897, 2011.

[18] J. M. Dietl and E. Garcia, "Stability in ornithopter longitudinal flight dynamics," Journal of Guidance, Control, and Dynamics, vol. 31, no. 4, pp. 1157-1163, 2008.

[19] H. Wagner, "Über die entstehung des dynamischen auftriebes von tragflügeln," ZAMM-Journal of Applied Mathematics and Mechanics/Zeitschrift für Angewandte Mathematik und Mechanik, vol. 5, no. 1, pp. $17-35,1925$.

[20] T. Theodorsen and W. Mutchler, "General theory of aerodynamic instability and the mechanism of flutter," 1935.

[21] I. E. Garrick, "Propulsion of a flapping and oscillating airfoil," 1937.

[22] H. Taha and A. S. Rezaei, "Viscous extension of potential-flow unsteady aerodynamics: the lift frequency response problem," Journal of Fluid Mechanics, vol. 868, pp. 141-175, 2019.

[23] R. Fernandez-Feria, "Linearized propulsion theory of flapping airfoils revisited," Physical Review Fluids, vol. 1, no. 8, pp. 1-14, 2016.

[24] R. Fernandez-Feria, "Note on optimum propulsion of heaving and pitching airfoils from linear potential theory," Journal of Fluid Mechanics, vol. 826, pp. 781-796, 2017.

[25] Z. J. Wang, "Insect flight: From Newton's law to neurons," Annual Review of Condensed Matter Physics, vol. 7, pp. 281-300, 2016.

[26] L. M. Milne-Thomson, Theoretical Aerodynamics. Dover Books on Aeronautical Engineering Series, Dover Publications, 1973.

[27] E. Akoz and K. W. Moored, "Unsteady propulsion by an intermittent swimming gait," Journal of Fluid Mechanics, vol. 834, pp. 149-172, 2018.

[28] C. Eloy, "Optimal strouhal number for swimming animals," Journal of Fluids and Structures, vol. 30, pp. 205-218, 2012.

[29] C. J. Pennycuick, Modelling the flying bird, vol. 5. Elsevier, 2008.

[30] G. K. Taylor, R. L. Nudds, and A. L. Thomas, "Flying and swimming animals cruise at a strouhal number tuned for high power efficiency," Nature, vol. 425, no. 6959, p. 707, 2003.

[31] A. Martín-Alcántara and R. Fernandez-Feria, "Assessment of two vortex formulations for computing forces of a flapping foil at high Reynolds numbers," Physical Review Fluids, vol. 4, no. 2, p. 024702, 2019.

[32] R. T. Jones, Wing Theory. Princeton: Princeton University Press, 1990.

[33] A. L. Thomas, "On the aerodynamics of birds' tails," Philosophical Transactions of the Royal Society of London. Series B: Biological Sciences, vol. 340, no. 1294, pp. 361-380, 1993.

[34] F. Fedoul, L. Parras, C. del Pino, and R. Fernandez-Feria, "Experimental study of the aerodynamic characteristics of a low-aspect-ratio flat plate array in a configuration of interest for a tidal energy converter," Journal of Fluids and Structures, vol. 48, pp. 487-496, 2014.

[35] S. Martínez-Aranda, A. García-González, L. Parras, J. VelázquezNavarro, and C. Del Pino, "Comparison of the aerodynamic characteristics of the NACA0012 airfoil at low-to-moderate reynolds numbers for any aspect ratio," International Journal of Aerospace Sciences, vol. 4, no. 1, pp. 1-8, 2016.

[36] C. Pennycuick, "Predicting wingbeat frequency and wavelength of birds," Journal of experimental biology, vol. 150, no. 1, pp. 171-185, 1990. 